\title{
Simultaneous Signal Acquisition by Synchronous Detection of Orthogonal Frequency Components
}

\author{
Matthias Baer ${ }^{1}$, Panayiota Demosthenous ${ }^{2}$, Bernhard Schmauss ${ }^{1}$ \\ ${ }^{1}$ Institute of Microwaves and Photonics (LHFT) and Erlangen Graduate School for Advanced Optical \\ Technologies (SAOT), Cauerstr. 9, 91058 Erlangen, Germany \\ 2 Cyprus Research \& Innovation Center Ltd (CyRIC), \\ 72, 28 ${ }^{\text {th }}$ Octovriou Ave, Off.301, Engomi, 2414 Nicosia, Cyprus \\ matthias.baer@fau.de
}

This paper is an outcome of the Project POST-DOC/0718/0186 which is co-funded by the European Regional Development Fund and the Republic of Cyprus through the Research and Innovation Foundation.

\section{Summary:}

We describe a multiplexing technique to simultaneously measure the absorption properties of gas at two different wavelengths using just one photodetector and an orthogonal frequency synchronous detection technique with non-sinusoidal modulation. This concept is demonstrated in a near infrared (NIR) spectroscopic application, measuring ethanol vapor in air.

Keywords: Near infrared, spectroscopy, integrating sphere, lock-in, synchronous detection

\section{Synchronous Detection and Integrating Spheres in Spectroscopy}

To gather even the smallest signal or signal change in spectroscopic applications, the usage of lock-in amplifiers is a commonly used technique. Synchronous detection, the principle behind lock-in amplifiers has been described in a historical overview already in 1954 [1]. However, some more detailed and application specific literature can be found in [2][3][4]. A principle schematic of a synchronous detector is depicted in Fig. 1. At this point, it shall be pointed out, that the reference signal does not necessarily need to be a sinusoidal signal, but can be more generally the excitation signal of the system.

Integration spheres are known to be beneficial in some spectroscopic applications, especially in scattered reflectance or transmittance measurements [5][6]. Especially integrating spheres are beneficial in gas sensing applications, as they easily increase the effective optical path length from the light source to the detector. Hence, the interaction length between light and gas sample becomes higher [7].

\section{Application and Optical Setup}

In the work presented here, an integrating sphere is used to measure ethanol vapor in air. The setup is sketched in Fig. 2. Two light sources (LS) are mounted to the sphere. The first LS is a DFB Laser with a wavelength of $2274 \mathrm{~nm}$, which is close to an absorption band of ethanol. The second LS is a NIR-LED at a wavelength of roughly $1550 \mathrm{~nm}$, which is intended to measure any other analyte but ethanol.

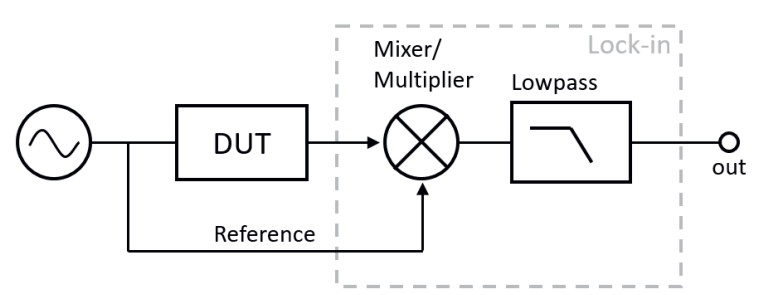

Fig. 1: Simple schematic of a synchronous detector.

The light of the LSs is detected with a photodetector (PD) which is sensitive for both wavelengths. The PD is mounted to a port of the integrating sphere that is not centered. Thus, no direct illumination of the PD by any of the LSs is possible. The port at which a sample can be mounted is actually at the rear side of the integrating sphere and thus not visible.

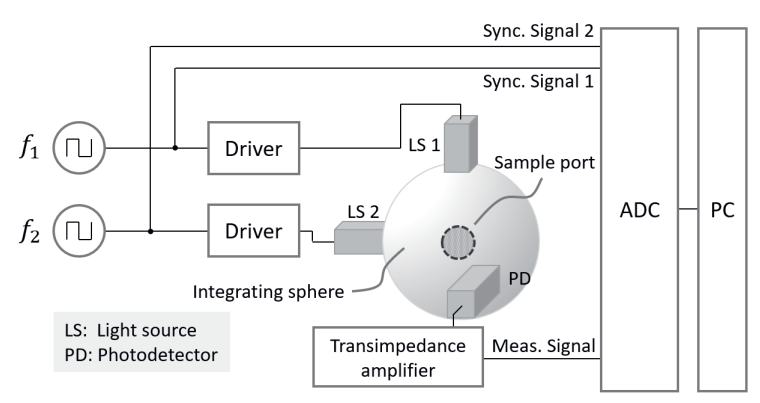

Fig. 2: Optical and electrical setup.

During measurement, both LSs are operated simultaneously and are pulsed with a rectangular waveform with a duty cycle of $50 \%$. While LS 1 is modulated with a frequency of $480 \mathrm{~Hz}$, LS 2 is modulated with a frequency of $985 \mathrm{~Hz}$, which is roughly the double frequency. It shall be emphasized that the ratio of these frequencies was chosen consciously. A synchronous 
detector with a rectangular waveform (duty cycle of $50 \%$ ) as reference signal does not only show a sensitivity at the fundamental frequency, but also at all odd harmonics, though with decreasing sensitivity. Thus care has to be taken, that the modulation signals of LS 1 and LS 2 do not share any harmonics. For the given case only odd harmonics are expected and by choosing the modulation frequencies with a ratio of two, the constraint of no common harmonics is fulfilled. Thus both signals are mathematically orthogonal to each other, which guarantees cross sensitivity freedom. However, in the considered case the frequency ratio is not exactly two and at some point two harmonics will come to lie at almost the same frequency. Fortunately, as mentioned before, the sensitivity of the synchronous detector decreases for higher harmonics, so that this problem is not severe.

The actual synchronous detection of the PD signal is done digitally by sampling the measurement and excitation signals (Sync. Signal 1/2) and processing them with a PC. Fig 3 shows snippets of the two synchronization signals and the measurement signal. It can be seen, that the measurement signal is a superposition of both excitation signals scaled by any factor.
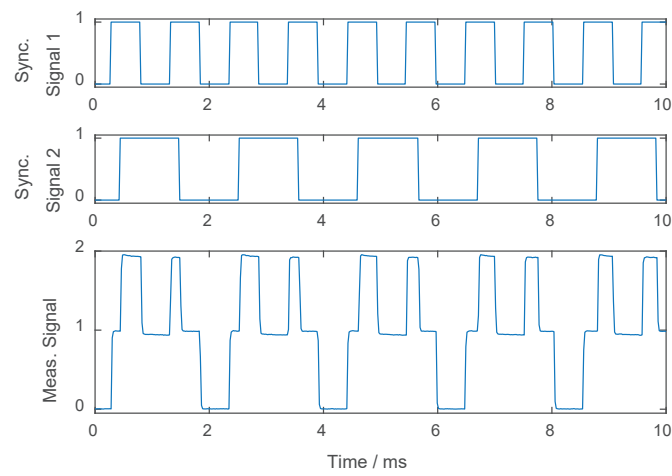

Fig. 3: Sampled signals according to Fig. 2.

Fig. 4 sketches the digitally implemented lock-in algorithm. With a rectangular excitation signal beeing used for both LSs, the algorithm is basically a multiplication of the measurement signal with the sign of the mean value free synchronization signal.

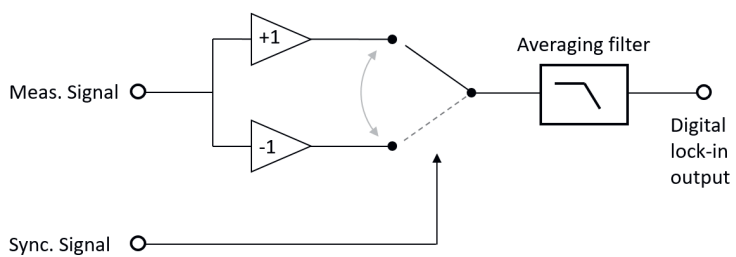

Fig. 4: Principle of the digitally implemented synchronous detector.

\section{Results}

A measurement (Fig. 5) has been performed by first flushing the integrating sphere with air. Then the sample port was left open for some time to measure the signal amplitude from both LSs at the detector when no analyte is present. After roughly 200 seconds, a cotton wool ball soaked with a $37.5 \%$ vol. ethanol solution was brought to the sample port. As the ethanol vapor fills the integration sphere, the detected intensity of LS1 decreases while the intensity of LS 2 is unaffected.

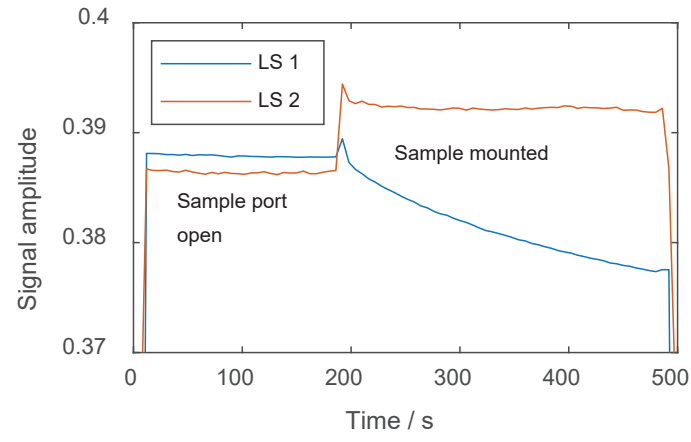

Fig. 5: Measured light intensity of LS 1 and LS 2 at the photodetector. The separation of both is done by using synchronous detection.

Simultaneous measurement of two different light intensities by orthogonalization of both signals using different modulation frequencies has been demonstrated.

\section{References}

[1] D. G. Tucker, "The history of the homodyne and synchrodyne," in Journal of the British Institution of Radio Engineers, vol. 14, no. 4, pp. 143-154, April 1954, doi: 10.1049/jbire.1954.0021.

[2] Bentham Instruments Ltd. Technical Note, Lock-in amplifiers, 2017, http://support.bentham.co.uk/support/ solutions/articles/13000036065-lock-in-amplifier-tutorial

[3] Zürich Instruments AG Whitepaper, Principles of lock-in detection and the state of the art, 2016, https://www.zhinst.com/sites/default/files/li_primer/zi_w hitepaper_principles_of_lock-in_detection.pdf

[4] Stanford Research Systems Application Note \#3, About Lock-In Amplifiers, https://www.thinksrs.com/ downloads/pdfs/applicationnotes/AboutLIAs.pdf

[5] Perkin Elmer Application Note, Applications and Use of Integrating Spheres With the LAMBDA 650 and 850 UV/Vis and LAMBDA 950 UV/Vis/NIR Spectrophotometers,

https://www.perkinelmer.com/CMSResources/Images/4 4-74191APP_LAMBDA650IntegratingSpheres.pdf

[6] Leonard M. Hanssen and Keith A. Snail, Integrating Spheres for Mid- and Near-infrared Reflection Spectroscopy, Chapter of "Handbook of Vibrational Spectroscopy", John Wiley \& Sons Ltd

[7] Sébastien Tranchart and Ikhlef Hadj Bachir and JeanLuc Destombes, Sensitive trace gas detection with near-infrared laser diodes and an integrating sphere, APPLIED OPTICS Vol. 35, No. 3620 December 1996, doi: 10.1364/AO.35.007070 